\title{
Pseudoprogression with Neoadjuvant Immunotherapy for Cutaneous Melanoma
}

\author{
Diogo Garcia $^{a} \quad$ Juliana Rodrigues Beal ${ }^{a} \quad$ Daniel Mamere Alvarez ${ }^{a}$ \\ Ricardo Silvestre e Silva Macarenco $^{b}$ Gustavo Schvartsman ${ }^{a}$ \\ aDepartment of Medical Oncology, Hospital Israelita Albert Einstein, São Paulo, Brazil;

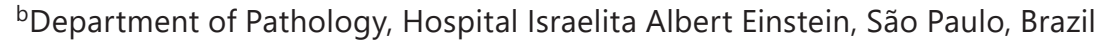

\author{
Keywords \\ Immunotherapy $\cdot$ Melanoma $\cdot$ PD-1 receptor $\cdot$ CTLA-4 receptor $\cdot$ RECIST
}

\begin{abstract}
Immune checkpoint inhibitors (ICl) have drastically changed the landscape of metastatic melanoma management, thus significantly improving survival. Clinically, assessing treatment response may be challenging in a portion of cases due to a massive influx of immune cells into the tumor microenvironment, causing a transient increase in the target lesion size. This phenomenon, coined pseudoprogression, can occur in $5-10 \%$ of metastatic patients, and it is commonly followed by a tumor regression. Its incidence, however, may be underestimated, given its ephemeral nature and often being documented in visceral metastatic lesions, which are only assessed by imaging scans every $2-3$ months. More recently, $\mathrm{ICl}$ has been studied in the neoadjuvant setting, yielding durable pathological responses in patients with cutaneous melanoma. Here, we report a case of a large retroauricular melanoma mass with regional lymph node involvement treated with ipilimumab and nivolumab combination therapy that developed pseudoprogression. Initially documented as an increase in size along with inflammatory features, followed by a dramatic clinical improvement. A complete regression was pathologically documented after 3 months and the patient remains disease-free for 14 months after treatment initiation. In conclusion, we document a pseudoprogression case during neoadjuvant $\mathrm{ICl}$ treatment and raise the question of whether the incidence of this phenomenon is higher when observed in superficial lesions, which can be assessed by routine physical exam.
\end{abstract}

\section{Karger $\stackrel{\text { !' }}{=}$}




\section{Introduction}

Management of metastatic melanoma has markedly improved with immune checkpoint inhibitors (ICIs), with 5-years overall survival exceeding 50\% with the combination of nivolumab and ipilimumab [1]. More recently, both programmed death-1 (PD-1) inhibitors nivolumab and pembrolizumab have been approved as adjuvant therapies for stage-III patients, paving the way for their administration in earlier stages of disease [2,3].

The combination of ipilimumab and nivolumab has been shown, to date, as the most promising neoadjuvant regimen for melanoma with macroscopic nodal invasion, with durable pathologic complete responses (pCR) in over half of cases, at a manageable toxicity profile when only 2 doses of ipilimumab at $1 \mathrm{mg} / \mathrm{kg}$ and nivolumab at $3 \mathrm{mg} / \mathrm{kg}$ were administered preoperatively [4].

Response assessment with ICI can often be challenging since a transient increase in target lesion size can occur in around $5-10 \%$ of the cases $[5,6]$. This phenomenon, coined pseudoprogression, is a result of a massive influx of immune cells and may be indicative of a response, despite an initial increase [7]. This finding has led to the development of the immune-related Response Criteria, which can better capture response patterns observed with some immunotherapeutic agents by allowing additional time on therapy after a first impression of disease progression if a clinical benefit is observed [8].

The true incidence of pseudoprogression, however, may be underestimated, given its ephemeral nature and the fact of often only being documented in visceral metastatic lesions, which are assessed by imaging scans every 2-3 months. In the neoadjuvant setting, with measurable disease by physical examination at every clinic visit, this event could be observed more frequently, and physicians should be familiar with this possible disease behavior after starting treatment. Here, we report a case of a patient undergoing neoadjuvant ipilimumab and nivolumab who developed a transient increase in tumor size that was documented by medical photography, causing concern of disease progression in both patient and the medical team.

\section{Case Report}

A 54-year-old man with no past medical history presented with a pigmented skin lesion in the left retro auricular area. He underwent an excisional biopsy in an outside facility that demonstrated a dermic epithelioid neoplasm with positive margins. Shortly afterward, he was evaluated at our service due to a recurrence arising from the resected scar tissue, now presenting as a large mass (Fig. 1a). The original biopsy specimen was reviewed and was consistent with melanoma, with mitotic rate of 2 mitosis $/ \mathrm{mm}^{2}$ and no angiolymphatic or perineural invasion. Information on Breslow thickness and ulceration was not available due to poor tissue quality. Staging with a PET-CT showed the left retroauricular mass, measuring $3.4 \times 3.0 \times 2.0 \mathrm{~cm}$, as well as multiple cervical lymph nodes (levels II, III, IV, V, and parotid) with signs of proximal facial nerve invasion, but no distant metastasis (AJCC 8th edition cT4aN3M0; Stage IIIC). A neoadjuvant strategy was preferred due to a high tumor burden, and the patient was started on ipilimumab at $1 \mathrm{mg} / \mathrm{kg}$ and nivolumab at $3 \mathrm{mg} / \mathrm{kg}$ every 3 weeks, with 2 cycles planned. After 4 days from the first dose, the retroauricular mass increased in size, with inflammatory features, causing worsening of his pain and a tingling sensation (Fig. 1b). On the seventh day, the inflammation had subsided and after 2 weeks the lesion started to decrease in size (Fig. 1c; see online suppl. Video 1; for all online suppl. material, see www.karger.com/doi/10.1159/000516036). Due to a good tolerance and an ongoing response, the patient completed 3 cycles of therapy. A repeat PET-CT showed a complete metabolic response. A surgical resection only of the underlying skin tumor bed was performed and a pathologic complete response (pCR) was observed (Fig. 2a-g).

\section{Karger'}




\section{Case Reports in Oncology}

\begin{tabular}{l|l}
\hline Case Rep Oncol 2021;14:881-885 \\
\hline DOI: 10.1159/000516036 & $\begin{array}{l}\text { ○ 2021 The Author(s). Published by S. Karger AG, Basel } \\
\text { www.karger.com/cro }\end{array}$ \\
\hline
\end{tabular}

Garcia et al.: Pseudoprogression with Immunotherapy for Melanoma
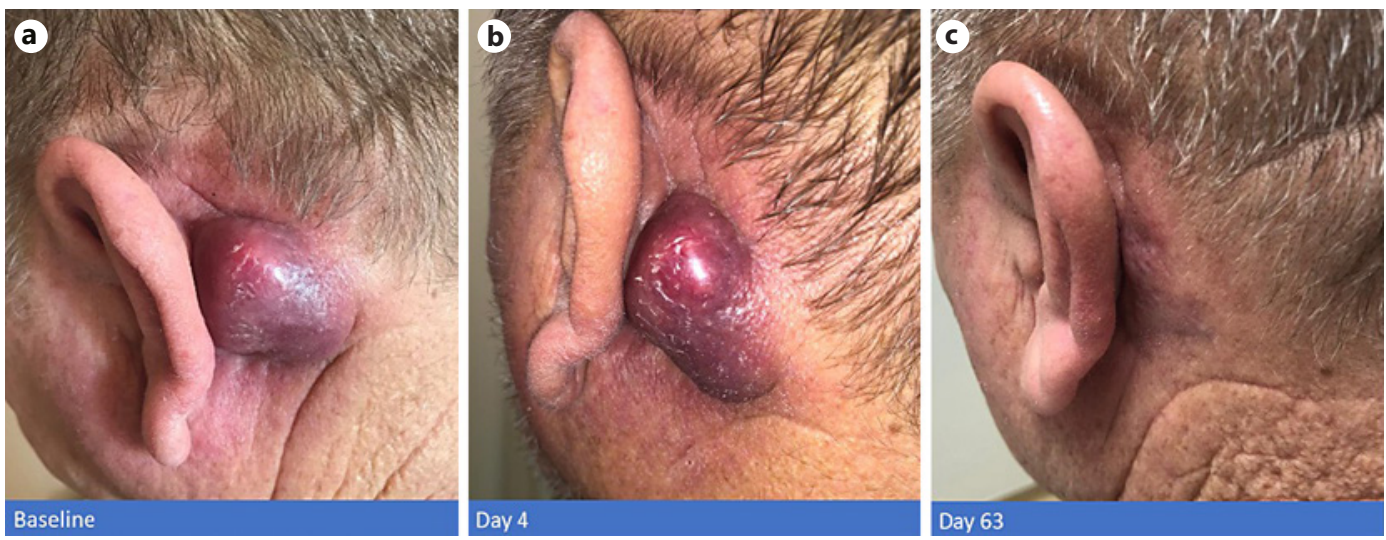

Fig. 1. Clinical response assessment after ipilimumab and nivolumab. Clinical evolution of response to immunotherapy in index lesion. Initial presentation of retro auricular mass (a); transient increase in lesion size, with inflammatory features and associated pain and tingling sensation (b); complete clinical response to immunotherapy after 3 cycles of ipilimumab plus nivolumab, later confirmed as a pCR (c). pCR, pathologic complete response.
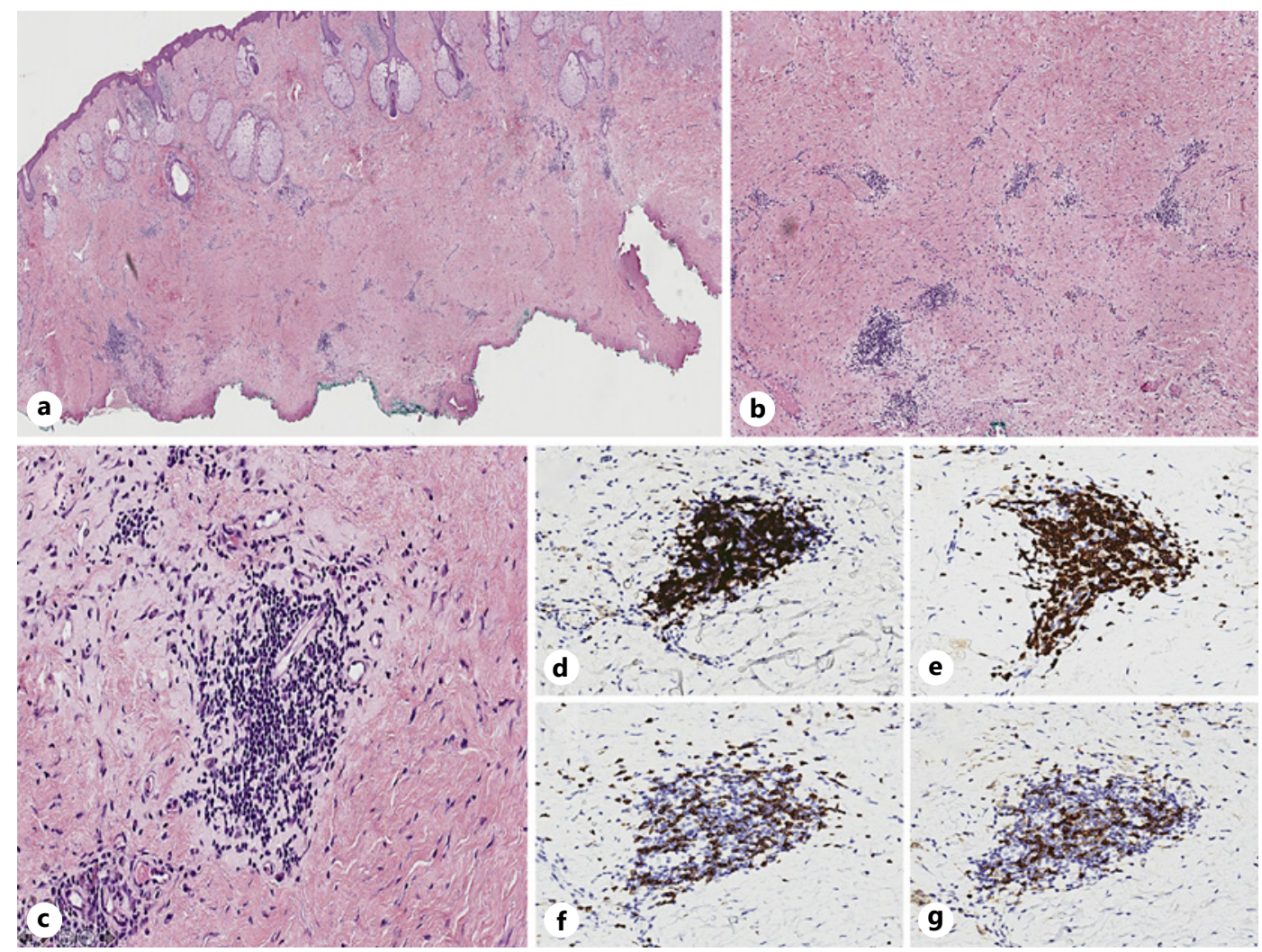

Fig. 2. Histopathologic slides from residual skin tumor bed. Histopathological sections revealed no residual melanocytic neoplasm, but deep dermal fibrosis and mild chronic lymphocytic inflammatory infiltrate. The latter was composed of mixed B-cell and T-cell populations, and T-cells presented hybrid CD4 and CD8 subpopulations. a Reticular dermis with fibrosis and no residual melanocytic neoplasm (HE. $\times 1$ ). b Reticular dermis with fibrosis and no residual melanocytic neoplasm (HE. $\times 4$ ). c Perivascular lymphocytic infiltrate $(H E, \times 14)$. d CD20-positive lymphocytes (HE. ×14). e CD3-positive lymphocytes (DAB. ×14). f CD4-positive lymphocytes (DAB. ×14). g CD8-positive lymphocytes (DAB. ×14). 
Neck lymph node dissection was deferred due to a high risk of facial nerve impairment. The patient is currently in surveillance with no evidence of disease after 14 months from treatment initiation.

\section{Discussion}

ICIs mediate immune response by either stimulating T-cell proliferation, activation, and differentiation or by suppressing the downregulating effect tumor cells can accomplish via inhibitory signals [9]. Immunotherapy has achieved outstanding results in melanoma at different stages of disease, but also brought atypical patterns of response, including pseudoprogression [10]. The phenomenon is defined as a $25 \%$ increase or more in tumor burden (including new lesions) that is not confirmed by the next imaging assessment [8]. Histologically, rather than tumor cell proliferation, pseudoprogression is characterized by immune infiltration, justifying its transient behavior.

Treatment with neoadjuvant combination immunotherapy for stage-III melanoma patients has shown promising results, with overall response rates above $70 \%$ and pCR rates and near-pCR rates of 42 and 62\%, respectively [11]. Adoption of a neoadjuvant approach allows physicians to follow visible and palpable lesions regularly and may lead to more frequent and earlier detection of pseudoprogression than the documented $10 \%$ incidence in visceral metastases, assessed only every 6-8 weeks by cross-sectional imaging. This was noted by the higher incidence of pseudoprogression described in the Optim trial, where $48 \%$ of patients who respond to intralesional Talimogene laherparepvec (T-Vec) had a transient increase in target lesion size, which could be explained both by the inflammatory features triggered by T-Vec and more frequently assessed cutaneous/subcutaneous/lymph node lesions [12].

Our patient achieved a pCR after 3 cycles of ipilimumab plus nivolumab. Due to the seemingly excellent prognosis of such patients [11], as well as the expected surgical morbidity, with a high risk of facial nerve injury, a consensus between medical team and patient was formed to defer total lymph node dissection. This strategy is being evaluated in the PRADO trial, where $60 \%$ of patients treated with ipilimumab and nivolumab who achieved a pCR/ near-pCR were spared from total lymph node dissection [13].

\section{Conclusion}

In conclusion, pseudoprogression is an infrequent pattern of response, introduced to our practice along with ICI. As neoadjuvant immunotherapy sets course for a new standard of care in locally advanced melanoma, it is important that patients and physicians be more familiarized with distinct response patterns, in order to anticipate outcomes and prevent unnecessary procedures. Additionally, we encourage that new estimates of the incidence of pseudoprogression be researched in the neoadjuvant setting.

\section{Statement of Ethics}

Written informed consent was obtained from the patient for publication of this case report and any accompanying images. The authors complied with internationally accepted standards for research practice and reporting.

\section{Karger'k}




\section{Conflict of Interest Statement}

G.S. declares consulting services for Bristol-Myers Squibb, Merck, Sharp \& Dome, AstraZeneca, Sanofi, Amgen, Roche, and Novartis.

\section{Funding Sources}

The authors did not receive any funding sources.

\section{Author Contributions}

All the authors made substantial contributions to the conception and design of the work; acquisition, analysis, and interpretation of data; drafting the work, and revising it critically for important intellectual content. All the authors approved the final version to be published. All the authors are in agreement to be accountable for all aspects of the work in ensuring that questions related to the accuracy or integrity of any part of the work are appropriately investigated and resolved.

\section{References}

1 Larkin J, Chiarion-Sileni V, Gonzalez R, Grob JJ, Rutkowski P, Lao CD, et al. Five-year survival with combined nivolumab and ipilimumab in advanced melanoma. N Engl J Med. 2019;381(16):1535-46.

2 Eggermont AMM, Blank CU, Mandala M, Long GV, Atkinson V, Dalle S, et al. Adjuvant pembrolizumab versus placebo in resected stage III melanoma. N Engl J Med. 2018;378:1789-801.

3 Weber J, Mandala M, Del Vecchio M, Gogas HJ, Arance AM, Cowey CL, et al. Adjuvant nivolumab versus ipilimumab in resected stage III or IV melanoma. N Engl J Med. 2017;377:1824-35.

4 Rozeman EA, Menzies AM, van Akkooi ACJ, Adhikari C, Bierman C, van de Wiel BA, et al. Identification of the optimal combination dosing schedule of neoadjuvant ipilimumab plus nivolumab in macroscopic stage III melanoma (OpACIN-neo): a multicentre, phase 2, randomised, controlled trial. Lancet Oncol. 2019;20:948-60.

5 Long GV, Weber JS, Larkin J, Atkinson V, Grob JJ, Schadendorf D, et al. Nivolumab for patients with advanced melanoma treated beyond progression: analysis of 2 phase 3 clinical trials. JAMA Oncol. 2017;3:1511-9.

6 Beaver JA, Hazarika M, Mulkey F, Mushti S, Chen H, He K, et al. Patients with melanoma treated with an antiPD-1 antibody beyond RECIST progression: a US food and drug administration pooled analysis. Lancet Oncol. 2018;19:229-39.

7 Hodi FS, Butler M, Oble DA, Seiden MV, Haluska FG, Kruse A, et al. Immunologic and clinical effects of antibody blockade of cytotoxic T lymphocyte-associated antigen 4 in previously vaccinated cancer patients. Proc Natl Acad Sci U S A. 2008;105:3005-10.

8 Wolchok JD, Hoos A, O’Day S, Weber JS, Hamid O, Lebbé C, et al. Guidelines for the evaluation of immune therapy activity in solid tumors: immune-related response criteria. Clin Cancer Res. 2009;15:7412-20.

9 Dougan M, Dranoff G. Immune therapy for cancer. Annu Rev Immunol. 2009;27:83-117.

10 Wolchok JD, Hoos A, O’Day S, Weber JS, Hamid O, Lebbé C, et al. Guidelines for the evaluation of immune therapy activity in solid tumors: immune-related response criteria. Clin Cancer Res. 2009;15(23):7412-20.

11 Menzies AM, Amaria RN, Rozeman EA, Huang AC, Tetzlaff MT, van de Wiel BA, et al. Pathological response and survival with neoadjuvant therapy in melanoma: a pooled analysis from the international neoadjuvant melanoma consortium (INMC). Nat Med. 2021;27(2):301-9.

12 Andtbacka RH, Ross M, Puzanov I, Milhem M, Collichio F, Delman KA, et al. Patterns of clinical response with talimogene laherparepvec (T-VEC) in patients with melanoma treated in the OPTiM phase III clinical trial. Ann Surg Oncol. 2016;23:4169-77.

13 Blank CU, Reijers ILM, Pennington T, Versluis JM, Saw RP, Rozeman EA, et al. First safety and efficacy results of PRADO: a phase II study of personalized response-driven surgery and adjuvant therapy after neoadjuvant ipilimumab (IPI) and nivolumab (NIVO) in resectable stage III melanoma. J Clin Oncol. 2020;38(15_Suppl l):10002. 